\title{
Coeficientes de utilización digestiva aparente de materia seca, proteína y aminoácidos esenciales de ingredientes terrestres para el camarón del Pacífico Litopenaeus vannamei (Decapoda: Penaeidae)
}

\author{
Martín Terrazas, Roberto Civera*, Lilia Ibarra \& Ernesto Goytortúa \\ 1. Laboratorio de Nutrición Acuícola. Centro de Investigaciones Biológicas del Noroeste (CIBNOR). Calle Mar Bermejo \\ \# 195, Col. Playa Palo de Santa Rita. La Paz, B. C. S., Código Postal 23090, México; terrazas@uabcs.mx, \\ * rcivera04@cibnor.mx, libarra04@cibnor.mx, netog04@ cibnor.mx \\ * Correspondencia.
}

Recibido 20-I-2010. Corregido 20-VI-2010. Aceptado 21-VII-2010.

\begin{abstract}
Apparent digestion coefficients for dry matter, protein and essential amino acids in terrestrial ingredients for Pacific shrimp Litopenaeus vannamei (Decapoda: Penaeidae). Protein quality mainly depends on the essential amino acid (EAA) profile, but also on its bioavailability, because EAA digestibility is generally lower than the analyzed amounts. This information is needed in the aquaculture industry for aquafeed formulation. For this purpose, the apparent digestibility coefficients of dry matter, protein, and essential amino acids of eight feedstuffs of terrestrial origin were determined for the juvenile whiteleg shrimp Litopenaeus vannamei (15-19g), using $1 \%$ chromic oxide as an inert marker. A reference diet was formulated and produced in the laboratory. Eight experimental diets were prepared each with $30 \%$ of one of the experimental ingredients added to the reference diet: casein, porcine byproduct meal poultry byproduct meal, corn meal, wheat gluten meal, soybean paste, sorghum meal, and wheat meal. The experiment consisted of a single-factor, completely randomized design with three replicates per treatment. Samples of ingredients, diets and feces were analyzed for nitrogen and amino acids. For amino acid assay, we used reverse-phase high performance liquid chromatography. To avoid partial loss of methionine and cystine, samples of ingredients, diets, and feces were oxidized with performic acid to methionine sulfone and cysteic acid prior to acid hydrolysis. The apparent dry matter and protein digestive utilization coefficients varied from $68 \%$ to $109 \%$ and from $70 \%$ to $103 \%$, respectively. Apparent digestibility of protein for casein, soy paste, wheat meal and wheat gluten were very high (over 90\%), corn gluten and poultry byproducts meal showed high protein digestibility (over $80 \%$ ), but porcine byproducts meal and sorghum meal had low digestibility ( $76 \%$ and $70 \%$, respectively). There was a reasonable, but not total, correspondence between apparent protein digestibility and average essential amino acid digestibility coefficients, except for arginine in corn gluten, phenylalanine and leucine in sorghum meal, phenylalanine in soy paste and lysine in wheat meal and poultry by-product meal. The most digestible feed ingredients for whiteleg shrimp were: wheat gluten, wheat meal and soy paste; poultry byproduct meal and corn gluten were less digestible and the lowest digestibility occurred in porcine byproduct meal and sorghum meal. Feedstuffs exhibited great variability in dry matter, protein and amino acid digestive utilization coefficients, which should be considered when formulating shrimp feeds. Rev. Biol. Trop. 58 (4): 1561-1576. Epub 2010 December 01.
\end{abstract}

Keywords: digestibility, dry matter, protein, amino acids, feedstuffs, shrimp.

La proteína es un nutrimento importante en la alimentación del camarón, ya que la velocidad de crecimiento de ese crustáceo en cultivo, depende de la cantidad de aminoácidos esenciales contenidos en los alimentos balanceados fabricados para su engorde (Tacon et al. 2002). Sin embargo, la proteína del alimento puede ser también el principal vector de contaminación ya que, a partir de ella se genera una gran cantidad de nitrógeno que es desechado en los cuerpos de agua con efectos negativos para el medio ambiente (Jackson et al. 2003). Una herramienta para reducir los costos de alimentación y la cantidad de nitrógeno desechado en 
los estanques, es la de optimizar los niveles de proteína en el alimento, lo cual se logra si se conoce el perfil de aminoácidos esenciales en las materias primas usadas en la fabricación de alimentos, ya que ello permite generar un mejor balance proteico y una mayor retención de nitrógeno en el organismo (Forster \& Dominy 2006).

Convencionalmente, la harina de pescado ha sido la principal fuente de proteína en la industria de alimentos para camarón, utilizando el $17.6 \%$ de la producción mundial para ese fin (Davis et al. 2004). La alta demanda comercial de esa harina ha reducido su disponibilidad comercial e incrementado su precio por lo que es recomendable el uso de ingredientes alternos para su sustitución. Existe información que indica la factibilidad de reemplazo total o parcial de la harina de pescado por otros ingredientes, tanto de origen animal como vegetal, tal es el caso de la harina de subproductos de aves (Cheng et al. 2002) y porcinos (Hernández et al. 2008) o la pasta de soya (Lim \& Dominy 1990) entre otros.

La combinación de las fuentes de proteína con las de energía (cereales) busca reflejar en el perfil de aminoácidos del alimento el de aminoácidos esenciales requeridos por el camarón (Tacon et al.2002), lo cual puede ser mejorado con la inclusión de aminoácidos cristalinos puros en el alimento (Wilson 2003, Forster \& Dominy 2006); por ejemplo, al usar pasta de soya como fuente de proteína se induce una deficiencia de metionina, por ello ya se estudia el uso de la metionina cristalina en la alimentación del camarón (Forster \& Dominy 2006).

La determinación de los coeficientes de utilización digestiva de materia seca, proteína y aminoácidos en los ingredientes es importante para obtener mayor precisión en el proceso de formulación, ya que considera qué porción de proteína y cada aminoácido está siendo digerida y potencialmente se encuentra disponible para el crecimiento del camarón, esto permite conocer a los insumos de mayor digestibilidad, generando alimentos más amigables para el medio ambiente. El objetivo del presente trabajo fue determinar los coeficientes de digestibilidad de materia seca, proteína y los aminoácidos esenciales en distintos ingredientes de origen terrestre empleados en la alimentación del camarón.

\section{MATERIALES Y MÉTODOS}

Ingredientes evaluados: Se utilizaron ocho ingredientes de origen terrestre para determinar su digestibilidad aparente in vivo de materia seca (DAMS), proteína (DAP) y aminoácidos esenciales (DAAA) utilizando el método indirecto de sustitución propuesto por Cho \& Slinger (1979). Los ingredientes de origen animal evaluados fueron: caseína (CAS), utilizada como control, harina de subproductos avícolas (HSPA) y harina de subproductos porcícolas (HSPP). Los ingredientes de origen vegetal fueron: harina de trigo (HT) y de sorgo (HS), pasta de soya (PS), gluten de maíz (GLM) y de trigo (GLT).

Los ingredientes fueron molidos en un pulverizador PULVEX 200 (Molinos Pulvex, D.F. México) y tamizados en una malla de $250 \mu \mathrm{m}$ (No. 35), con excepción de la HSPP, que únicamente fue tamizada a $500 \mu \mathrm{m}$, ya que su elevado contendido de grasa no permitió su paso por un tamiz de menor diámetro.

Formulación y fabricación de alimentos: Los alimentos se formularon con la ayuda del paquete Brill Formulation (V.1.35.003, Feed Management System, Fairmont, MN, USA). Se fabricaron 9 dietas en total: una dieta de referencia, formulada para contener aproximadamente $35 \%$ de proteína cruda (Cuadro 1) y 8 dietas de prueba conteniendo $70 \%$ (p/p en húmedo) de la dieta de referencia y $30 \%$ de los ingredientes a evaluar. Se utilizó $1 \%$ de óxido crómico $\left(\mathrm{Cr}_{2} \mathrm{O}_{3}\right)$ como marcador inerte. Los ingredientes secos fueron homogenizados en una mezcladora de cocina (Kitchen Aid MR, St. Joseph, MI, USA) antes de adicionar una emulsión preparada por separado, conteniendo lecitina de soya y aceite de pescado, de acuerdo a lo recomendado por Civera \& Guillaume (1989). Después de que la emulsión fue agregada y mezclada, se adicionó agua $\left(35^{\circ} \mathrm{C}\right)$ a un volumen de $30 \%$ del peso de los ingredientes 
CUADRO 1

Composición proximal ( $\mathrm{g} / 100 \mathrm{~g}$ peso seco) y contenido de energía de los ingredientes evaluados

TABLE 1

Proximate composition ( $\mathrm{g} / 100 \mathrm{~g}$ dry matter) and gross energy content of the tested ingredients

\begin{tabular}{|c|c|c|c|c|c|c|}
\hline Ingrediente & $\mathrm{PC}(\%)$ & $\mathrm{EE}(\%)$ & $\mathrm{FC}(\%)$ & $\mathrm{C}(\%)$ & $\operatorname{ELN}^{1}(\%)$ & Energía bruta $(\mathrm{cal} / \mathrm{g})$ \\
\hline \multirow[t]{2}{*}{ Caseína $^{2}$} & 91.2 & 0.0 & 0.8 & 0.7 & 7.3 & 3761 \\
\hline & \pm 0.3 & \pm 0.0 & \pm 0.1 & \pm 0.1 & & \pm 4 \\
\hline \multirow[t]{2}{*}{ Harina de subproductos avícolas ${ }^{3}$} & 65.0 & 12.4 & 0.1 & 13.7 & 8.8 & 4671 \\
\hline & \pm 0.2 & \pm 0.2 & \pm 0.0 & \pm 0.0 & & \pm 15 \\
\hline \multirow[t]{2}{*}{ Harina de subproductos de porcícolas ${ }^{4}$} & 57.3 & 9.5 & 0.5 & 25.1 & 7.6 & 4286 \\
\hline & \pm 0.1 & \pm 0.4 & \pm 0.0 & \pm 0.0 & & \pm 8 \\
\hline \multirow[t]{2}{*}{ Harina de trigo ${ }^{5}$} & 12.7 & 0.7 & 0.2 & 0.5 & 85.9 & 3766 \\
\hline & \pm 0.1 & \pm 0.1 & \pm 0.0 & \pm 0.4 & & \pm 5 \\
\hline \multirow[t]{2}{*}{ Harina de sorgo ${ }^{6}$} & 8.4 & 3.9 & 1.4 & 1.6 & 84.7 & 4035 \\
\hline & \pm 0.1 & \pm 0.0 & \pm 0.2 & \pm 0.0 & & \pm 5 \\
\hline \multirow[t]{2}{*}{ Pasta de soya ${ }^{7}$} & 52.9 & 2.6 & 2.0 & 6.95 & 35.5 & 4282 \\
\hline & \pm 0.0 & \pm 0.1 & \pm 0.15 & \pm 0.04 & & \pm 5 \\
\hline \multirow[t]{2}{*}{ Gluten de maíz ${ }^{8}$} & 72.3 & 2.5 & 0.4 & 1.5 & 23.2 & 5337 \\
\hline & \pm 0.2 & \pm 0.0 & \pm 0.0 & \pm 0.0 & & \pm 2 \\
\hline \multirow[t]{2}{*}{ Gluten de trigo ${ }^{9}$} & 83.2 & 1.5 & 0.3 & 0.8 & 14.2 & 5036 \\
\hline & \pm 0.2 & \pm 0.0 & \pm 0.0 & \pm 0.0 & & \pm 4 \\
\hline
\end{tabular}

$\mathrm{PC}=$ proteína cruda, $\mathrm{EE}=$ extracto etéreo, $\mathrm{FC}=$ fibra cruda, $\mathrm{C}=$ cenizas. Valores promedio de 3 réplicas \pm desviación estándar. ${ }^{1}$ Extracto libre de nitrógeno $=100 \%$ - (\%proteína cruda $+\%$ extracto etéreo+\%fibra cruda+\%cenizas $) .{ }^{2}$ SIGMA C-3400. ${ }^{3}$ Harina de subproductos de pollo y pavo (Ind. Griffind. Jackson Mi. USA). ${ }^{4}$ CONAGRA. Iowa, USA. ${ }^{5}$ Harinera Parayas, S.A. de C.V., Jalisco, México. ${ }^{6}$ Valle de Santo Domingo, B.C.S., México. ${ }^{7}$ AGYDSA. Jalisco, México. ${ }^{8}$ EURONUTEC, Queretáro, Qro., México. ${ }^{9}$ PROBST S.A. de C.V., Tlalneplanta, Edo. de México, México.

mezclados. La masa maleable y dúctil resultante fue extruida utilizando un molino de carne (Torrey, MJ12, Monterrey, NL., México) con un dado de $2 \mathrm{~mm}$. Los pelets fueron secados en una estufa con aire forzado a $45^{\circ} \mathrm{C}$ de temperatura hasta alcanzar una humedad aproximada de $10 \%$. Los alimentos terminados se mantuvieron en refrigeración hasta su uso en el bioensayo de digestibilidad.

Sistema experimental. Se realizó un bioensayo de digestibilidad en el Laboratorio de Nutrición Experimental del CIBNOR, que cuenta con acuarios de fibra de vidrio con fondo color gris y una capacidad de $60 \mathrm{~L}$ $(50 \times 55 \times 38 \mathrm{~cm})$. Cada uno está equipado con una malla mosquitero para evitar la fuga de organismos, un calentador sumergible de $200 \mathrm{w}$ (Ebo-Jager, Eheim GmbH \& Co., KG Deizisau,
Alemania) ajustables a una precisión de $\pm 0.5^{\circ} \mathrm{C}$, un sistema de drenaje para el recambio de agua y limpieza, además de un exhaustor externo para airear el agua con mangueras alimentadas por un soplador de $5 \mathrm{HP}$ y con ello conseguir valores de oxígeno disuelto iguales o mayores a $5 \mathrm{mg} / \mathrm{L}$. Los acuarios se abastecieron con el agua de mar (36-40\%o de salinidad) iniciando en una toma a $300 \mathrm{~m}$ de la orilla del mar con una bomba de 15 HP que envía el agua a una cisterna externa con capacidad de $240 \mathrm{~m}^{3}$; posteriormente el agua se envía hacia otra cisterna de $5 \mathrm{~m}^{3}$ pasando por un filtro de arena de $70 \mu \mathrm{m}$ (Cristal-Flo, Modelo T240BP1 Santa Rite Industries Inc., Delavan, WI, USA). El agua se bombeó hacia los acuarios, pasando a través de filtros de cartucho $(10$ y $5 \mu \mathrm{m})$ y luz ultravioleta. Los acuarios contaron con un 
sistema de iluminación de luz de neón de 200w y el control artificial del fotoperíodo se realizó por medio de un reloj automático. El fotoperíodo utilizado fue de $12 \mathrm{~h}$ de luz constante a partir de las 6:00hrs.

Organismos experimentales: Los camarones usados en el presente trabajo fueron juveniles de la especie Litopenaeus vannamei (Bonne, 1931), entre 15-19g, obtenidos en la granja Acuacultores de la Península (APSA) ubicada al Norte de la ciudad de La Paz, B.C.S., México. Antes de iniciar el experimento, los animales se mantuvieron en tanques con capacidad de 2500 L por cuatro meses en el Laboratorio de Nutrición Experimental del CIBNOR. Los organismos se alimentaron dos veces al día (10:00 y 16:00hrs) con un alimento comercial para camarón (35\% de proteína en base húmeda) hasta que alcanzaron el peso deseado para iniciar el bioensayo (media 16.5; desv. estándar $1.04 \mathrm{~g}$ ).

Determinación de la digestibilidad in vivo: Antes de iniciar el ensayo de digestibilidad in vivo, los camarones fueron transferidos a los acuarios rectangulares de 60 litros a una densidad de 4 organismos por acuario y fueron aclimatados a los alimentos experimentales durante una semana antes de iniciar la recolecta de heces. Las 9 dietas fueron evaluadas por triplicado (acuario) durante 45 días en promedio. Diariamente se monitorearon la temperatura $\left(27 \pm 1^{\circ} \mathrm{C}\right)$ con un termómetro de mercurio, la salinidad $(39 \pm 1 \%$ o $)$ por medio de un refractómetro portátil (Vista A366 ATC) y el oxígeno disuelto $(5.0 \pm 0.3 \mathrm{mg} / \mathrm{mL})$ con un oxímetro portátil (YSI, 550A, Yellow Springs, OH, USA). Los camarones fueron alimentados tres veces al día (10:00, 14:00 y 18:00h).

Cada día por la mañana (7:30) el alimento no consumido, exoesqueletos de las mudas y heces excretadas durante la noche fueron retiradas de los acuarios por sifoneo, realizando un recambio del $60 \%$ del agua en los acuarios. Una vez limpios los acuarios, se suministró el alimento (10:00hrs) correspondiente al 10\% de la biomasa de los camarones; al paso de dos horas se llevó a cabo la primera recolecta de las heces en cada acuario. Después de esta primera recolecta, se volvió a proporcionar alimento (14:00hrs), para luego de una hora proceder a una segunda ronda de recolecta, realizando recorridos para evitar que las heces permanecieran en el agua por más de una hora. La recolecta de las heces se llevó a cabo mediante sifoneo con la ayuda de una manguera de plástico $(0.5 \mathrm{~cm}$ de diámetro) unida a un tubo de vidrio en la punta. El material fecal de cada acuario fue recolectado en un recipiente de plástico, enjuagado suavemente en agua destilada para eliminar sales, congelado a $-90^{\circ} \mathrm{C}$, liofilizado y homogenizado con la ayuda de una espátula, a fin de contar con una sola muestra por acuario (réplica biológica). Posteriormente, las heces fueron analizadas por triplicado para determinar sus contenidos de proteína, aminoácidos y óxido crómico como se indica en la sección de análisis químicos.

Criterios de evaluación: Los coeficientes de digestibilidad aparente de la materia seca (DMS), de proteína (DAP) y aminoácidos (DAAA) de la dieta de referencia (Cuadro 1) fueron calculados usando las siguientes ecuaciones (Cho \& Slinger 1979):

$\% \mathrm{DMS}=100-100$

(\% $\mathrm{Cr}$ en el alimento/\% Cr en las heces)

$$
\% \mathrm{DAP}=100-100
$$

$\% \mathrm{Cr}$ en el alimento/\% $\mathrm{Cr}$ en las heces)x (\% PC heces/\%PC en alimento)

$$
\% \text { DAAA }=100-100
$$

(\% Cr en el alimento/\%Cr en las heces)X (\%AA heces $/ \%$ AA en alimento)

Donde: Cr=óxido crómico.

Para el caso de los ocho ingredientes evaluados (IE), la digestibilidad aparente de la materia seca (DMS), la proteína (DAP) y los aminoácidos esenciales (DAAA) se calcularon usando las siguientes ecuaciones (Bureau \& Hua 2006): 


$$
\begin{aligned}
& \% \mathrm{DAMS}=\mathrm{DAMS}_{\text {dieta de prueba }}{ }^{+} \\
& {\left[\left(\mathrm{DAMS}_{\text {dieta de prueba }}-\mathrm{DAMS}_{\text {dieta de referencia }}\right)\right.} \\
& \left.\mathrm{x}\left(\left(0.7 \times \mathrm{xMS}_{\text {dieta de referencia }}\right) /\left(0.3 \times \mathrm{MS}_{\mathrm{IE}}\right)\right)\right] \\
& \% \text { DAPIE }=\mathrm{DAP}_{\text {dieta de prueba }}{ }^{+} \\
& {\left[\left(\mathrm{DAP}_{\text {dieta de prueba }}-\mathrm{DAP}_{\text {dieta de referencia }}\right)\right.} \\
& \left.\mathrm{x}\left(\left(0.7 \times \mathrm{xMS}_{\text {dieta de referencia }}\right) /\left(0.3 \times \mathrm{MS}_{\mathrm{IE}}\right)\right)\right] \\
& \% \text { DAAATi=DAAAE } \text { dieta de prueba }^{+} \\
& {\left[\left(\mathrm{DAAA}_{\text {dieta de prueba }}-\mathrm{DAAA}_{\text {dieta de referencia }}\right)\right.} \\
& \left.\mathrm{x}\left(\left(0.7 \times \mathrm{MS}_{\text {dieta de referencia }}\right) /\left(0.3 \times \mathrm{MS}_{\mathrm{IE}}\right)\right)\right]
\end{aligned}
$$

Donde: IE=ingrediente evaluado; $\mathrm{MS}=\%$ de materia seca en la dieta.

Análisis químicos: Se analizaron por triplicado los ingredientes y los alimentos experimentales. El análisis químico proximal fue realizado según las metodologías recomendadas por la A.O.A.C. (2005). La humedad se determinó por secado en estufa a $70^{\circ} \mathrm{C}$ durante $24 \mathrm{~h}$. La cuantificación de cenizas (C) o minerales (método 942.05), se desarrolló por incineración de las muestras en una mufla (Thermolyne modelo F6000, Dubuque, IA, USA) a $550^{\circ} \mathrm{C}$ durante 6h. El análisis de proteína cruda (PC) (método 2001.11), se realizó con la técnica micro Kjeldahl. La medición de extracto etéreo (EE) (método 2003.05), se hizo mediante un sistema de extracción (Soxtec Avanti modelo 2050, Foss Tecator, Höganäs, Suiza), usando como solvente éter de petróleo. Para determinar la fibra cruda (FC) (método 978.10), se usó una hidrólisis ácido-básica con ácido sulfúrico e hidróxido de sodio en un digestor (Fibertec modelo M6, Foss Tecator, Höganäs, Suiza). Se calculó el extracto libre de nitrógeno (ELN), por diferencia de 100 con los porcentajes de las determinaciones anteriores. La concentración de proteína se determinó en las excretas recolectadas en el bioensayo de digestibilidad usando la metodología descrita anteriormente (A.O.A.C. 2005).

Energía bruta: Para la determinación de energía bruta de ingredientes y alimentos se usó un calorímetro adiabático (Parr Instrument Co., Modelo 1261, Moline, IL), para ello se utilizaron crisoles de acero inoxidable en donde se colocaron pastillas de $1 \mathrm{~g}$ previamente secadas a $70^{\circ} \mathrm{C}$ en una estufa por $12 \mathrm{~h}$, se empleó alambre $(10 \mathrm{~cm})$ con una aleación de niquel-cobre el cual estaba en contacto con la muestra, la muestra fue incinerada en una cámara de combustión inyectada con oxígeno. Posteriormente, los residuos líquidos de la combustión fueron recuperados y titulados con carbonato de sodio $0.725 \mathrm{~N}$; naranja de metilo fue empleado como indicador, también se cuantificó la cantidad de alambre calcinado durante la combustión para los cálculos de la energía liberada.

Análisis de aminoácidos: Para el caso de los aminoácidos esenciales (excepto triptófano) y cistina (no esencial), se analizaron por triplicado muestras de los ingredientes, dietas y heces, usando cromatografía líquida de alta resolución (HPLC fase-reversa) como los describe Wu et al. (1997). Las muestras fueron preparadas de conformidad con el método 994.12 de la A.O.A.C. (2005) utilizando un equipo Hewlett Packard serie 1100. Para evitar pérdidas de metionina y cistina en las muestras durante la hidrólisis ácida (Wathelet 1999), éstas fueron previamente oxidadas con ácido perfórmico por $16 \mathrm{~h} \mathrm{a} 4^{\circ} \mathrm{C}$ bajo agitación constante. La metionina fue cuantificada como metionina sulfona y la cistina como ácido cisteíco (Baker 1997). Para la hidrólisis ácida, se adicionaron $3 \mathrm{ml}$ de $\mathrm{HCl} 6 \mathrm{~N} / \mathrm{fenol}$ a viales ámbar de $10 \mathrm{ml}$, se agitaron por 15 minutos, se engargolaron con sellos metálicos y se colocaron en una estufa previamente calentada a $110^{\circ} \mathrm{C}$ para su hidrólisis por $24 \mathrm{~h}$ (A.O.A.C. 2005). El contenido de $\mathrm{Cr}_{2} \mathrm{O}_{3}$ en los alimentos y heces fue determinado utilizando el método de digestión (Furukawa \& Tsukahara 1966).

Para los coeficientes de digestibilidad se utilizó el análisis de varianza de una vía, con el fin de determinar diferencias significativas entre los tratamientos y una posterior comparación de medias de Tukey (Steel \& Torrie 1996) con un nivel de confianza del $95 \%$. Para el procesamiento de los datos se utilizó el software de estadística SAS 1999, (V.8, SAS Institute Inc., Cary, NC. USA). 


\section{RESULTADOS}

Composición proximal, contenido de energía bruta y aminoácidos de los ingredientes: Los valores obtenidos del análisis proximal y energía bruta de los ocho ingredientes expresados en base seca, se muestran en el Cuadro 1. Los valores de proteína cruda más elevados se encontraron en la CAS (91.2\%), seguido por GLM y GLT con $72.3 \%$ y $83.2 \%$; mientras que PS, HSPA y HSPP tuvieron valores de $52.9 \%, 57.3 \%$ y $65.0 \%$, respectivamente. Los dos cereales analizados (HS, 8.4\%; HT, $12.7 \%$ ) mostraron los valores más bajos de proteína. En el caso de la fibra cruda, la HS y la PS resultaron con los valores más altos (1.4\% y $2.0 \%)$, el resto de los ingredientes tuvieron valores menores a $0.81 \%$. El contenido de extracto etéreo fue mayor en los dos ingredientes de origen animal (HSPA, 12.4\%; HSPP, 9.5\%), el HS resultó con un mayor nivel
(3.9\%) de extracto etéreo que lo encontrado en HT $(0.74 \%)$; GLM y PS presentaron valores similares $(2.5 \%$ y $2.6 \%)$, GLT mostró un $0.74 \%$ y CAS un $0.03 \%$, siendo éstos los más bajos.

Por otra parte, se encontró un alto contenido de cenizas en las harinas de HSPP, 25.1\% y HSPA $13.7 \%$; PS presentó un valor intermedio $(6.9 \%)$; mientras que HS y GLM tuvieron contenidos bajos y similares entre sí (1.62\% y $1.49 \%$ ). El GLT, HT y CAS mostraron un cantidad menor al $0.8 \%$.

Los valores de energía más alta se encontraron en los dos glútenes evaluados (maíz y trigo); mientras que la energía determinada en HS, PS, HSPP y HSPA fluctuó entre 4035 y 4672cal/g; HT mostró el menor contenido energético.

El perfil de aminoácidos esenciales (excepto triptófano) y cistina (no esencial) de los ingredientes se puede observar en el Cuadro 2. La concentración de aminoácidos analizada

CUADRO 2

Perfil de aminoácidos (\% en base húmeda) de los ingredientes evaluados

TABLE 2

Amino acid profile (\% as fed) of the tested ingredients

$\begin{array}{lcccccccccc}\text { Ingrediente } & \text { Met } & \text { Cis }^{1} & \text { His } & \text { Tre } & \text { Arg } & \text { Val } & \text { Fen } & \text { Ile } & \text { Leu } & \text { Lis } \\ \text { Caseína }^{2} & 2.67 & 0.54 & 2.65 & 3.60 & 3.08 & 5.75 & 4.65 & 4.75 & 8.26 & 6.18 \\ & \pm 0.43 & \pm 0.05 & \pm 0.13 & \pm 0.15 & \pm 0.16 & \pm 0.25 & \pm 0.16 & \pm 0.13 & \pm 0.41 & \pm 1.68 \\ \text { Harina de subproductos } & 1.24 & 0.53 & 1.27 & 2.59 & 4.71 & 2.72 & 2.10 & 2.62 & 4.14 & 2.93 \\ \text { avícolas }^{3} & \pm 0.02 & \pm 0.01 & \pm 0.14 & \pm 0.14 & \pm 0.29 & \pm 0.14 & \pm 0.10 & \pm 0.14 & \pm 0.25 & \pm 0.81 \\ \text { Harina de suproductos }^{3} & 0.77 & 0.45 & 0.98 & 1.66 & 4.10 & 2.44 & 1.87 & 1.85 & 3.31 & 2.14 \\ \text { porcícolas }^{4} & \pm 0.02 & \pm 0.05 & \pm 0.06 & \pm 0.02 & \pm 0.01 & \pm 0.31 & \pm 0.11 & \pm 0.02 & \pm 0.01 & \pm 0.56 \\ \text { Harina de trigo }^{5} & 0.18 & 0.23 & 0.28 & 0.30 & 0.49 & 0.57 & 0.54 & 0.51 & 0.77 & 0.43 \\ & \pm 0.01 & \pm 0.06 & \pm 0.01 & \pm 0.02 & \pm 0.01 & \pm 0.01 & \pm 0.01 & \pm 0.02 & \pm 0.00 & \pm 0.00 \\ \text { Harina de sorgo }^{6} & 0.14 & 0.14 & 0.17 & 0.25 & 0.43 & 0.47 & 0.47 & 0.34 & 1.15 & 0.61 \\ & \pm .0 .01 & \pm 0.04 & \pm 0.01 & \pm 0.02 & \pm 0.36 & \pm 0.12 & \pm 0.21 & \pm 0.10 & \pm 0.54 & \pm 0.01 \\ \text { Pasta de soya }^{7} & 0.59 & 0.55 & 1.36 & 1.76 & 3.47 & 2.26 & 2.47 & 2.17 & 3.58 & 2.73 \\ & \pm 0.00 & \pm 0.07 & \pm 0.08 & \pm 0.13 & \pm 0.19 & \pm 0.18 & \pm 0.23 & \pm 0.19 & \pm 0.33 & \pm 0.75 \\ \text { Gluten de maíz }^{8} & 2.04 & 1.08 & 1.32 & 1.98 & 1.94 & 2.87 & 4.08 & 2.76 & 9.83 & 1.18 \\ & \pm 0.05 & \pm 0.01 & \pm 0.03 & \pm 0.01 & \pm 0.01 & \pm 0.00 & \pm 0.02 & \pm 0.03 & \pm 0.07 & \pm 0.06 \\ \text { Gluten de trigo } & 1.25 & 1.61 & 1.61 & 1.93 & 2.58 & 3.17 & 4.32 & 3.16 & 5.28 & 1.23 \\ & \pm 0.02 & \pm 0.04 & \pm 0.09 & \pm 0.07 & \pm 0.08 & \pm 0.11 & \pm 0.22 & \pm 0.14 & \pm 0.20 & \pm 0.16\end{array}$

Valores promedio de 3 réplicas \pm desviación estándar.

Met=metionina, Cis=cistina $\left({ }^{1}\right.$ No esencial $)$, His=histidina, Tre=treonina, Arg=arginina, Val=valina, Fen=fenilalanina, $\mathrm{Ile}=$ isoleucina, $\mathrm{Leu}=$ leucina, $\mathrm{Lis}=$ lisina . 
CUADRO 3

Composición de ingredientes ( $\mathrm{g} / 100$ dieta en base húmeda) de la dieta de referencia

TABLE 3

Ingredient composition ( $\mathrm{g} / 100 \mathrm{~g}$ diet as fed) of the reference diet

\begin{tabular}{|c|c|}
\hline Harina de pescado (sardina Monterrey) ${ }^{1}$ & 33.60 \\
\hline Harina de trigo $^{2}$ & 30.11 \\
\hline Pasta de soya ${ }^{3}$ & 20.00 \\
\hline Aceite de pescado (sardina) $)^{4}$ & 4.00 \\
\hline Almidón de maíz ${ }^{5}$ & 3.50 \\
\hline Ácido algínico (aglutinante) ${ }^{6}$ & 2.00 \\
\hline Lecitina de soya ${ }^{7}$ & 2.00 \\
\hline Premezcla de vitaminas ${ }^{8}$ & 1.80 \\
\hline Fosfato dibásico de sodio ${ }^{9}$ & 1.20 \\
\hline Óxido crómico ${ }^{10}$ & 1.00 \\
\hline Premezcla de minerales ${ }^{11}$ & 0.50 \\
\hline Cloruro de colina ( $62 \%$ de agente activo $)^{12}$ & 0.20 \\
\hline Stay-C (vitamina $C, 35 \%$ activa $)^{13}$ & 0.09 \\
\hline Butil-hidroxi-tolueno (BHT) ${ }^{14}$ & 0.004 \\
\hline Total & 100.00 \\
\hline
\end{tabular}

1 Conservera San Carlos, Puerto San Carlos, B.C.S., Mexico). ${ }^{2}$ Harinera Parayas, S.A. de C.V., Jalisco, México. ${ }^{3}$ AGYDSA. Jalisco, México. ${ }^{4}$ Planta Procesadora San Carlos, B.C.S., México. ${ }^{5}$ SIGMA \# cat. S-4126. ${ }^{6}$ SIGMA \# cat. A-0503-1. ${ }^{7}$ Restaurante vegetariano Rey Sol, La Paz, B.C.S., México. 8 (ICN Biomedicals Inc. Ohio. USA, Sigma Co. St. Louis. USA). ${ }^{9}$ SIGMA-ALDRIH \# cat. S0876. ${ }^{10}$ IMPEX \# cat. $12233101162 .{ }^{11}$ ICN \# cat. ICN101386. ${ }^{12}$ ICN \# cat. ICN101386. ${ }^{13}$ ROCHE, D. F., México. ${ }^{14}$ ICN \# cat. 101162. Premezcla de vitaminas (UI o mg kg -1 dieta): Acetato vit. A, 15000 IU; D3, 7500 IU; E, 400; K3, 20; cloruro de colina (99\%) 400; tiamina $\mathrm{HCl}$, 150; riboflavina, 10; piridoxina $\mathrm{HCl}, 50$; ácido pantoténico, 100; niacina, 300; biotina, 1; inositol, 500; ácido fólico, 20; cianocobalamina, 0.1. ${ }^{11}$ Premezcla mineral $\left(\mathrm{mg} 100 \mathrm{~g}^{-1}\right.$ diet): $\mathrm{Na}_{2} \mathrm{HPO}_{4}, 2370 ; \mathrm{MgSO}_{4} \cdot 7 \mathrm{H}_{2} \mathrm{O}, 500 ; \mathrm{ZnSO}_{4} \cdot 7 \mathrm{H}_{2} \mathrm{O}$, 90; KCl, 500; $\mathrm{MnCL}_{2} \cdot 4 \mathrm{H}_{2} \mathrm{O}, 23.4 ; \mathrm{CuCL}_{2} \cdot 2 \mathrm{H}_{2} \mathrm{O}, 5 ; \mathrm{KI}$, 5; $\mathrm{CoCl}_{2} \cdot 6 \mathrm{H}_{2} \mathrm{O}, 2.5$.

es de acuerdo a lo esperado y consistente para un mismo grupo de ingredientes (harinas de pescado) ya que en lo general, a mayor cantidad de proteína mayor cantidad de cada uno de los aminoácidos. Los valores más altos se encontraron en Leu (8.3\%) y $\operatorname{Arg}(5.7 \%)$ en la caseína y Leu $(5.3 \%)$ en GLT; mientras que las cantidades más bajas fueron medidas en los aminoácidos azufrados (Met y Cis) en las harinas de trigo y sorgo. Por otra parte, en el Cuadro 3 se detalla la composición de los ingredientes de la dieta de referencia, y en el Cuadro 4 se presenta el análisis químico proximal de la dieta de referencia y las de prueba. La composición proximal, así como la de los perfiles de aminoácidos de las dietas de prueba son los esperados, ya que son el resultado de la combinación de $70 \%$ de la dieta de referencia con $30 \%$ de cada uno de los ingredientes de prueba.

Digestibilidad aparente de materia seca (DAMS), proteína (DAP) y aminoácidos (DAAA): Los coeficientes de DAMS y DAP de los 8 ingredientes evaluados se muestran en el Cuadro 5. En el caso de la DAMS, el menor y el mayor valor se encontraron en HSPP (68.2\%) y GLT (109.2\%); DAMS en ambos insumos y en CAS (97.3\%) fue mayor a la de los demás ingredientes $(\mathrm{p}<0.05)$. La HSPP y HT mostraron coeficientes similares con 89.0 y $89.4 \%$, respectivamente ( $\mathrm{p}>0.05)$. El GLM, HS y PS fueron similares entre sí, con 82.8, 82.4 y $85.4 \%(\mathrm{p}>0.05)$, respectivamente, mientras que PS fue igual a HSPA y HT ( $>>0.05)$. En lo que corresponde a digestibilidad de proteína, el GLT mostró el valor más alto (103.1\%), siendo similar HT, PS y CAS (100.3, 100.0 y $99.9 \%$, respectivamente). Estos valores fueron mayores a los de HSPA, GLM, HSPP y HS (88.3, 81.2, 75.8 y $69.9 \%$, respectivamente), que también fueron diferentes entre sí $(\mathrm{p}<0.05)$.

Los coeficientes de DAAA fluctuaron entre $61.6 \%$ para His en HS y $108.4 \%$ para Arg en el GLT (Cuadro 6). Los menores coeficientes se encontraron para Met, His, Tre, Val y Lis en HS (61.6 a 71.6\%), siendo similares ( $\mathrm{p}>0.05)$ a Fen y Lis en HSPP. Valores entre 70 y $76 \%$ se obtuvieron para Fen (GLM), Cis, Arg e Ile en HS; Cis y Val en HSPP; y Leu y Lis en GLM.

Valores similares $(p>0.05)$ cercanos al 80 y $83 \%$ se observaron para Val (GLM); His (HSPA); His, Ile y Leu (HSPP); Fen y Leu en HS; Tre (GLM), Cis (HSPA), Tre y Arg (HSPP) y Lis (HT). Coeficientes de AAAD alrededor de $88 \%$, se encontraron para Met, Cis e Ile en GLM; Tre, Val, Fen, Ile y Leu en HSPA; Fen 


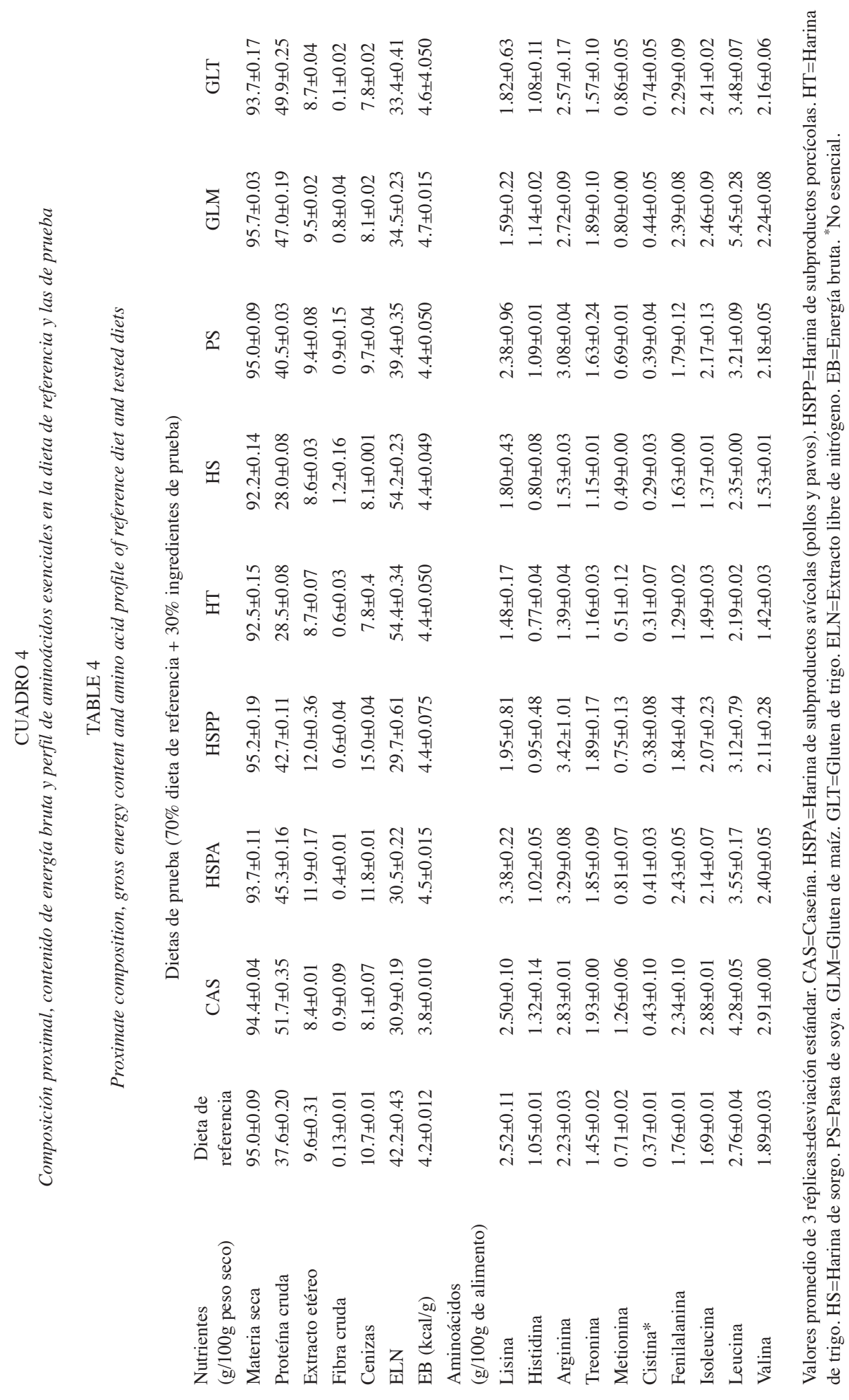




\section{CUADRO 5}

Coeficientes de utilización digestiva aparente de materia seca y proteína de los ingredientes evaluados

TABLE 5

Apparent digestibility coefficienst for dry matter and protein in the tested ingredients

$\begin{array}{lcc}\text { Ingrediente } & \text { DAMS } \% & \text { DAP }^{1} \% \\ \text { Caseína } & 97.3 \pm 3.0^{\mathrm{b}} & 99.9 \pm .5^{\mathrm{a}} \\ \text { Harina de subproductos avícolas } & 89.0 \pm 3.8^{\mathrm{c}} & 88.3 \pm 0.5^{\mathrm{b}} \\ \text { Harina de subproductos porcícolas } & 68.2 \pm 2.4^{\mathrm{e}} & 75.8 \pm 0.8^{\mathrm{d}} \\ \text { Harina de trigo } & 89.4 \pm 1.8^{\mathrm{c}} & 100.3 \pm 2.2^{\mathrm{a}} \\ \text { Harina de sorgo } & 82.4 \pm 2.7^{\mathrm{d}} & 69.9 \pm 3.4^{\mathrm{e}} \\ \text { Pasta de soya } & 85.4 \pm 1.7^{\mathrm{cd}} & 100.0 \pm 0.6^{\mathrm{a}} \\ \text { Gluten de maíz } & 82.8 \pm 1.6^{\mathrm{d}} & 81.2 \pm 0.4^{\mathrm{c}} \\ \text { Gluten de trigo } & 109.2 \pm 3.8^{\mathrm{a}} & 103.1 \pm 0.7^{\mathrm{a}}\end{array}$

${ }^{1}$ DAMS y DAP=digestibilidad aparente de materia seca y digestibilidad aparente de la proteína.

Valores promedio de 3 réplicas \pm desviación estándar.

En cada columna, medias con literales distintas son diferentes $(\mathrm{p}<0.05)$.

para PS y Met (HSPP). Valores cercanos a 93\% se detectaron para His (HSPA) y Tre (PS), siendo similares entre sí ( $\mathrm{p}>0.05)$.

Coeficientes entre 93.5 y $99.7 \%$ se obtuvieron para Tre y Leu de CAS; Arg en GLM; Arg y Lis en HSPA; Met, Cis, Val, Ile y Lis en PS; Tre y Fen en GLT; Cis, His, Arg, Val, Fen e Ile en HT. Valores próximos al $100 \%$ fueron detectados para Met, Cis, His, Arg, Val, Fen, Ile y Lis en CAS; His, Arg y Leu en PS; Met, Cis, His, Val, Ile, Leu y Lis en GLT, y Met y Tre en HT.

\section{DISCUSIÓN}

La caseína empleada aquí presentó una cantidad similar de proteína, mayor contenido de fibra, pero menor de lípidos y cenizas que la caseína reportada por Mu et al. (2000). Esto se puede deber al origen y al proceso de elaboración del ingrediente, sin embargo, el contenido de aminoácidos encontrado en CAS fue similar al perfil reportado previamente (DEGUSSA 2006). La caseína es un ingrediente semipurificado, al cual se le han eliminado la vitaminas durante el proceso de elaboración y es comúnmente utilizado como fuente de proteína para estudios nutricionales ya que tiene un elevado contenido de proteína y aminoácidos altamente digestibles (Tacon 1989), aunque no se utiliza para la fabricación de alimentos para acuacultura debido a su elevado costo y a que es deficiente en arginina y lisina para los organismos acuáticos.

Las cantidades de PC y EE en la harina de subproductos de ave del presente estudio, fue similar y menor, respectivamente, que los valores reportados por Thompson et al. (2008), mientras que los contenidos de FC y de cenizas fueron menores a los valores encontrados por Cheng et al. (2002), mientras que la energía fue similar a la reportada por estos últimos autores. Asimismo, Cheng et al. (2002) reportaron en una HSPA, una mayor cantidad de Met, Lis, Ile e His, una cantidad similar de Arg, Fen, Val y menores valores de Leu, Tre y Val que en la harina evaluada aquí.

Por su parte, Hernández et al. (2008) encontraron una composición proximal similar en la HSPP a la analizada en este estudio. El perfil de aminoácidos en HSPP analizada aquí, expresado como porcentaje de la proteína, fue mayor al perfil reportado por Hernández et al. (2008). La harina de trigo experimental mostró una cantidad de proteína similar, con menores cantidades de EE y cenizas que los valores 


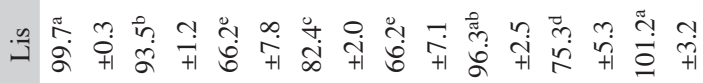

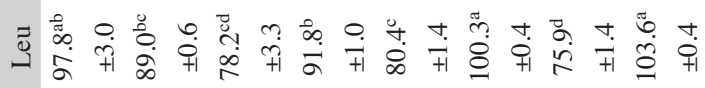

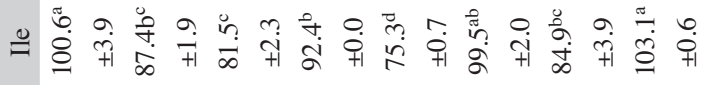

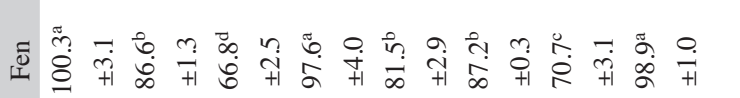

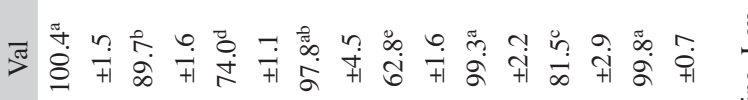

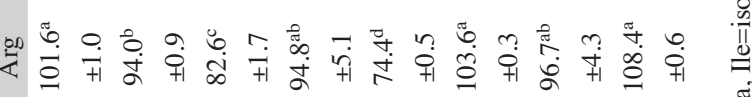

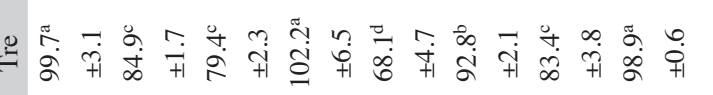

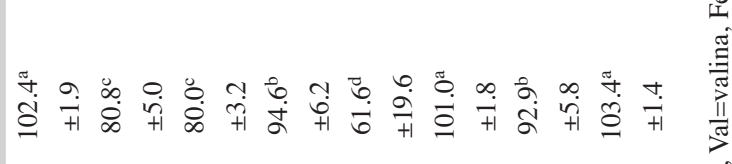

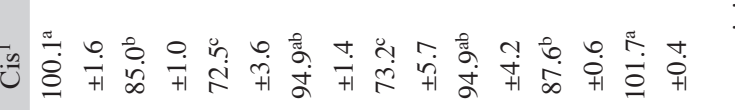

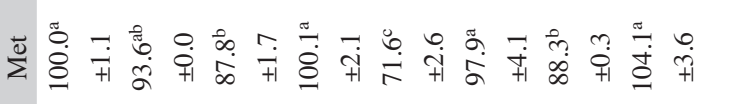


encontrados en el estudio realizado por Gatlin III et al. (2007).

La composición proximal de la HS tuvo cantidades similares de PC y EE que las reportadas por el NRC (1998), el contenido de cenizas fue muy parecido a los valores encontrados en cuatro variedades de sorgo por MariscalLandín et al. (2004). La harina de sorgo estudiada aquí, tuvo un $10 \%$ menos de energía que la encontrada por Rawles \& Gatlin III (2000). $\mathrm{El}$ grano de sorgo fue finamente molido y tamizado, y esto reduce el contenido de fibra cruda e incrementa la proteína. No obstante, la harina tuvo cantidades muy bajas de proteína y aminoácidos con respecto a los análisis reportados en otros sorgos (Dale 1995, NRC 1998).

La pasta de soya analizada presentó contenidos similares de PC, cenizas y EB, mayor cantidad de EE y menor de FC, que las cantidades reportadas por Fagbenro \& Davies (2001) para ese mismo ingrediente. La cantidad de aminoácidos mostrado en la PS fue más alto que el publicado en otro reporte (Sudaryono et al. 1999), lo cual es consistente con el mayor contenido de proteína cruda y menor de fibra cruda en la PS estudiada aquí.

El gluten de maíz del presente estudio tuvo un contenido mayor de PC que el encontrado por otros autores (Tibbetts et al. 2004, Kaur \& Saxena 2005) mientras que su contenido de EE, FC y energía fueron menores que los encontrados previamente (Tibbetts et al. 2004). Por el contrario, la cantidad de FC en GLM reportada por Kaur \& Saxena (2005) fue muy alta con respecto a lo encontrado aquí. El GLM mostró un contenido mayor de aminoácidos, consistente con su mayor contenido de proteína cruda, al ser comparado con lo publicado previamente (NRC 1994). Esto se debe a que los ingredientes evaluados fueron tamizados antes del bioensayo de digestibilidad y con ello se redujo el contenido de fibra cruda, resultando en una mayor concentración de proteína.

La composición proximal del gluten de trigo estudiado aquí tuvo un mayor nivel de proteína y EE, con menores cantidades de FC y cenizas, que el mismo ingrediente evaluado por Lazo et al. (1998). La harina de trigo experimental mostró una cantidad de proteína similar, con menores cantidades de EE y cenizas, que los valores encontrados en otro estudio (Gatlin III et al. 2007). Los resultados obtenidos en proteína cruda y contenido de aminoácidos en los productos de trigo (harina y gluten) de este estudio fueron similares a los encontrados en otras tablas de composición química de ingredientes (DEGUSSA 2006).

La digestibilidad aparente de materia seca de los diferentes ingredientes evaluados presentó diferencias significativas $(\mathrm{p}<0.05)$, obteniéndose valores superiores al $80 \%$ en casi todos ellos con excepción de la harina de subproductos porcícolas, que fue inferior a $69 \%$. El ingrediente que mostró mejor digestibilidad fue el gluten de trigo seguido de la caseína. Considerando que la DAMS puede ser útil para estimar la cantidad de material no digerible de un ingrediente o alimento, los ingredientes que mejor fueron aprovechados por el camarón fueron el gluten de trigo y la caseína.

Los valores de DAMS obtenidos con el gluten de maíz y con la HSPA son superiores a los reportados por Yang et al. (2009). Brunson et al. (1997) reportan valores de DAMS para la PS, la HT y la HS inferiores a los obtenidos en el presente trabajo, por su parte Cruz-Suárez et al. (2009) reportaron valores similares para la PS y la HSPA estudiadas aquí. Para el caso de HSPP, existe información que indica (Hernandez et al. 2008) que DAMS en una dieta para juveniles de camarón blanco con $25 \%$ de HSSP fue de $81 \%$, mientras que DAP fue de $85 \%$; esos valores fueron más altos que los encontrados en el presente estudio, sin embargo, la información de esos mismos autores indica que conforme se incrementan los niveles de HSPP en el alimento, DAMS y DAP disminuyen. Esto concuerda con el hecho de que DAMS es afectada por altos contenidos de cenizas y fibra cruda (Sudaryono et al. 1999) por lo que es razonable pensar que la HSPP presente una DAMS tan baja (68.2\%) con respecto a las demás, ya que es el ingrediente que presenta mayor cantidad de cenizas, lo cual era de esperarse dado que contiene carne y huesos de cerdo. Asimismo, es posible que 
el tamaño de partícula de la harina de subproductos de porcinos $(500 \mu \mathrm{m})$ haya disminuido su digestibilidad, ya que se ha reportado que a mayor tamaño de partícula la digestibilidad de un ingrediente generalmente es menor (Tacon 1989). Observando los valores aquí obtenidos, podemos suponer que el efecto de la fibra cruda y las cenizas sobre la DAMS está en función de su cantidad en el ingrediente, ya que HSPA presenta una DAMS similar $(89.0 \%)$ o superior a la de otros ingredientes con contenidos de cenizas y fibra cruda inferiores. Akiyama et al. (1993) recomiendan valores máximos de cenizas y fibra de 15 y $4 \%$ respectivamente en los alimentos para camarón, ya que un exceso de estos compuestos tiende a disminuir la digestibilidad.

Para el caso de la harinas de cereales, HS presentó menores coeficientes de digestibilidad (DAMS y DAP) que los encontrados en HT; esto podría deberse en parte a que el sorgo resultó con 6 veces más fibra y 4 veces más cenizas que las contenidas en la harina de trigo, ya que ambos factores han sido relacionados con una menor digestibilidad (Yang et al. 2009) y por el hecho que, los ingredientes fibrosos tienen una tasa de pasaje mayor por el tracto digestivo del camarón siendo expuestos por menos tiempo a las enzimas digestivas (Lazo et al. 1998). Asimismo, la estructura del almidón del sorgo presenta una mayor concentración de amilosa que de amilopectina con respecto al trigo (Mckevith 2004); siendo la amilosa de mayor densidad lo que dificulta su gelatinización y por ende resultando en una menor digestión tanto de carbohidratos, como de la proteína (Cruz-Suárez et al. 1994). Por otra parte, algunas variedades de sorgo son altas en taninos, los cuales interfieren con la actividad enzimática al precipitar la fracción proteica de las mismas, decreciendo la digestibilidad del propio cereal o de otros ingredientes en un mismo alimento. Sin embargo, esto no explicaría nuestros resultados en virtud de que el sorgo utilizado en el este trabajo poseía una testa sin pigmentos, lo que supone la ausencia o baja cantidad de taninos en ese cereal (Mckevith 2004).
Los valores de DAMS obtenidos con el gluten de trigo son superiores a los reportados por Yang et al. (2009), pero similares a los reportados por Brunson et al. (1997). Los valores de digestibilidad encontrados para materia seca y proteína del gluten de trigo fueron superiores al $100 \%$. Altos coeficientes de digestibilidad han sido explicados parcialmente por problemas de lixiviación de nutrimenos en los alimentos (Cruz-Suárez et al. 2009), sin embargo, esta posibilidad es remota ya que la dieta de prueba con $30 \%$ de GLT presentó la mayor estabilidad en el agua (resultados no mostrados aquí).

Convencionalmente, es de suponer que los coeficientes de digestibilidad fluctúen entre 0 y $100 \%$, pero hasta ahora no se ha determinado completamente el por qué en ocasiones se generan resultados con digestibilidades mayores al $100 \%$. Sin embargo, valores negativos (Akiyama et al. 1989) o mayores al $100 \%$ en la digestibilidad medida con camarones no son inusuales, ya que han sido reportados en otros estudios (Divakaran et al. 2000, Cruz-Suárez et al. 2001, Rivas-Vega et al. 2009).

Cruz-Suárez et al. (2009) sugieren que la digestibilidad en algunos aminoácidos, como en el caso de la lisina y la metionina, pueden ser sobreestimados debido a su alta solubilidad en el agua marina. Coeficientes de digestibilidad inesperados se pueden obtener debido a interacciones desconocidas entre ingredientes o al contenido de material endógeno en las heces, como puede ser la membrana peritrófica (Akiyama et al. 1989). Valores de $110 \%$ en la digestibilidad de la proteína en el gluten de trigo con el camarón P. setiferus fueron reportados por Brunson et al. (1997), ellos atribuyeron esa elevada digestibilidad a posibles interacciones entre los nutrimentos de los ingredientes. Otra razón que puede afectar la correcta determinación de la digestibilidad, puede estar relacionada con las elevadas temperaturas que en ocasiones son utilizadas durante la fabricación de algunos ingredientes, ya que se ha demostrado que se origina un daño en la estructura de los aminoácidos afectando su digestibilidad (reacción de Maillard); dependiendo de la 
intensidad de la reacción, algunos aminoácidos pueden ser eliminados en la orina sin ser utilizados a nivel celular para la síntesis de proteína (Sibbald 1987, Terrazas et al. 2005). Por ello, los aminoácidos que han sido alterados por altas temperaturas pueden contribuir a sobreestimar los valores de digestibilidad si no son analizados en muestras de orina. Liou et al. (2005) desarrollaron una técnica para la recolecta de orina en camarones afin de determinar el contenido de aminoácidos, sin embargo, es un método poco práctico.

La proteína es el ingrediente más crítico en los alimentos para camarón, tanto desde el punto de vista de costo, como de la respuesta de crecimiento del organismo, además, juega un papel muy importante desde el punto de vista ecológico, ya que la proteína proveniente del alimento es una de las principales fuentes de nitrógeno en los estanques de cultivo, provocando eutroficación. Para el caso de la digestibilidad aparente de proteínas, el GLT, y HT, PS y CAS presentaron valores significatimente superiores $(\mathrm{p}<0.05)$ a los demás ingredientes, siendo el grano de sorgo el que presentó un valor significativamente inferior al resto de los ingredientes.

Akiyama et al. (1989) reportaron que la DAP de ingredientes semi-purificados o concentrados, es superior a la de los no purificados, lo cual se pudo observar en el presente trabajo al comparar los valores obtenidos entre el gluten y el grano de trigo (103.1 y $100.3 \%$ respectivamente). Los bajos valores de DAP obtenidos con HSPP y HS (75.8 y 69.9\% respectivamente) pueden estar relacionados, para el caso de HSPP con su alto contenido en cenizas.

Con relación a la DAMS y DAP del GLT y GLM, la mejor digestibilidad encontrada en GLT posiblemente no sea explicada por la cantidad de fibra cruda $(0.44 \%)$ y cenizas (1.49\%) en GLM, ya que ambas son el doble de lo encontrado en GLT (ya que esos valores son relativamente bajos) por lo que es poco probable suponer que se pueda aplicar la regla de que a mayor cantidad de ambas fracciones una menor digestibilidad (Yang et al. 2009). Sin embargo, la diferencia en la digestibilidad entre los nutrimentos evaluados en GLT y GLM sea mejor explicada por la cantidad del extracto libre de nitrógeno, ya que esa fracción es 39\% mayor en GLM y en ella se encuentra almidón el cual posee una relación de amilosa con respecto a la amilopectina similar a la del grano de sorgo, cuya estructura posiblemente afecte negativamente la digestibilidad (Cruz-Suárez et al. 1994). De manera similar a lo reportado por Akiyama et al. (1989), en el presente trabajo los valores de DAP no forzosamente fueron influenciados por el origen del ingrediente, esto es, animal o vegetal.

Los valores de digestibilidad aparente de aminoácidos esenciales presentaron un comportamiento similar al de DAP, donde los valores más altos se presentaron con GT, CAS, PS y HT, mientras que los valores más bajos se presentaron con HSPP y HS. Esto resulta lógico ya que la calidad o disponibilidad de la proteína presente en un ingrediente está en función de su contenido en aminoácidos. Yu (2006) estudió la DAAA de HSPA en dos especies de camarón (P. monodon y L. vannamei) $\mathrm{y}$ en términos generales, la DAMS, DAP y DAAAD para Arg, Val y Cis fueron menores a los obtenidos en el presente trabajo. El mismo autor reportó coeficientes mayores para His, Ile, Fen y Tre, e iguales para Met, Leu y Lis con respecto a lo obtenido aquí. La DAAA de todos los aminoácidos evaluados en la HSPP fueron menores con rangos entre 10 y $20 \%$ con respecto a lo reportado por Yu et al. (2006a).

En lo que corresponde a la DAAA del sorgo usado en este estudio, los resultados indicaron una similitud en Met, Ile y Leu, con valores más altos para His, Lis, Fen, Tre y Val, y menores en Arg, que los reportados en la tilapia del Nilo según Guimarães et al. (2008). En un trabajo donde se evaluaron distintos lotes de pasta de soya (Akiyama et al. 1991) se obtuvieron coeficientes de DAAA menores en 9 aminoácidos (excepto Lis el cual fue similar), con respecto a los valores obtenidos en el presente estudio. Por su parte Akiyama et al. (1989) también reportaron digestibilidades menores de aminoácidos con respecto a los 
coeficientes estudiados en el presente trabajo, con excepción de Fen.

La información generada en este trabajo permitirá desarrollar procesos de formulación de alimentos balanceados para el camarón blanco L. vannamei con ingredientes más amigables con el medio ambiente y aportar datos necesarios para el desarrollo de trabajos de investigación con el fin de determinar los requerimientos nutricionales del camarón blanco con base en proteínas y aminoácidos digestibles. Los resultados obtenidos en este trabajo demuestran que existen diferencias marcadas en los coeficientes de utilización digestiva aparente de la materia seca, de proteína y los aminoácidos esenciales entre los ingredientes terrestres evaluados. La variablidad observada en la digestibilidad de los ingredientes es debida principalmente a diferencias en la estructura química y los métodos de procesamiento. El gluten de trigo, la harina de trigo, pasta de soya y harina de subproductos avícolas fueron los mejor utilizados, por lo que se considera que son mejores opciones para la formulación y fabricación de alimentos balanceados para juveniles del camarón blanco.

\section{AGRADECIMIENTOS}

A Mariela Camacho por su capacitación en la determinación de aminoácidos con el equipo HPLC. A Margarita Herrera, Armando Reyes y Sandra de La Paz por su apoyo en el bioensayo y la recolecta de heces. A Sonia Rocha y Dolores Rondero por los análisis proximales. A la compañía Malta Cleyton de México por el financiamiento parcial del trabajo, así como al proyecto AC 2.4 del CIBNOR. Al CONACYT por la beca de doctorado para Martín Terrazas.

\section{RESUMEN}

Los aminoácidos esenciales y su disponibilidad son importantes en la formulación de alimentos. La digestibilidad de materia seca (DAMS), proteína (DAP) y aminoácidos esenciales (DAAA) fueron determinados (triplicado) para el camarón blanco del Pacífico, Litopenaeus vannamei (15-19g), usando una dieta de referencia con 30\% (de cada ingrediente). Los ingredientes evaluados fueron: caseína (CAS), harinas de subproductos avícolas (HSPA) y porcícolas (HSPP), gluten de maíz (GLM) y trigo (GLT), pasta de soya (PS), harinas de sorgo (HS) y trigo (HT). La DAMS y DAP variaron entre 68\%-109\% y 70\%-103\%, respectivamente. La DAP en CAS, PS, HT y GLT fue mayor al $90 \%$, en GLM y HSPA superior a $80 \%$; HSPP (76\%) y HS (70\%) tuvieron menor digestibilidad. Hubo concordancia entre DAP y DAAA, excepto para Arg en GLM, Fen y Leu en HS, Fen en PS y Lis en HT y HSPA. Se encontró una gran variabilidad en la DAMS, DAP y DAAA en los ingredientes, lo que debe ser tomado en cuenta al formular alimentos para camarón.

Palabras clave: digestibilidad, materia seca, proteína, aminoácidos, ingredientes, camarón.

\section{REFERENCIAS}

Akiyama, D.M., S.R. Coelho, A.L Lawrence \& E.H. Robinson. 1989. Apparent digestibility of feedstuffs by the marine shrimp Penaeus vannamei BOONE. Nippon Suisan Gakkaishi. 55: 91-98.

Akiyama, D.M. 1991. Soybean meal utilization by marine shrimp, p. 207-225. In D.M. Akiyama \& R.K.H Tan (eds.). Proceedings of the aquaculture feed processing and nutrition workshop. Tailandia-Indonesia, September 19-25.

Akiyama D.M. 1993. Semi-extensive shrimp farm management. ASA Technical Bulletin, MITA (P) No.518/12/92, Vol. AQ. 38 1993/3. American Soybean Association, Singapur, Singapur.

A.O.A.C. 2005. Official methods of analysis of AOAC International. Amino acids in feeds, p. 8-24. In W. Horwitz \& G. Latimer (eds.). Association of Analytical Chemists. Gaithersburg, Maryland, Virginia, EEUU.

Baker, D.H. 1997. Ideal amino acid profiles for swine and poultry and their applications in feed formulation. FERMEX. Technical Review-9. Nutri-Quest, Inc. Chesterfield, Missouri, EEUU.

Brunson, J.F. \& R.P Romaire. 1994. Digestibilidad de ingredientes alimenticios para el camarón blanco Penaeus setiferus, p. 231-233. In R. Mendoza Alfaro, L.E. Cruz-Suárez \& D. Ricque-Marie (eds.). Memorias del II Simposium Internacional de Nutrición Acuícola. 7-9 Noviembre. Monterrey, Nuevo León, México.

Brunson, J.F., R.P. Romaire \& R.C. Reigh. 1997. Apparent digestibility of selected ingredients in diets for white shrimp Penaeus setiferus L. Aquac. Nutr. 7: 9-16.

Bureau, D.P. \& K. Hua. 2006. Letter to the editor of Aquaculture. Aquaculture 252: 103-105. 
Civera, R. \& J.C. Guillaume. 1989. Effect of sodium phytate on growth and tissue mineralisation of Penaeus japonicus and Penaeus vannamei juveniles. Aquaculture 77: 145-156.

Cheng, Z.J., K.C. Behnke \& W.G. Dominy. 2002. Effects of poultry by-product meal as a substitute for fish meal in diets on growth and body composition of juvenile pacific white shrimp Litopenaeus vannamei. J. Appl. Aquaculture 12: 71-83.

Cruz- Suárez, L.E., D. Ricque-Marie, J.D. Pinal-Mansilla \& P. Wesche-Ebelling. 1994. Effect of different carbohydrate sources on the growth of Penaeus vannamei: economical impact. Aquaculture 123: 349-360.

Cho, C.Y. \& S. Slinger. 1979. Apparent digestibility measurement in feedstuffs for raibow trout, p. 239-247. In J.E. Halver \& K. Tiews (eds.). Finfish Nut. and Tech. Vol. II. Heenemann GmbH, Berlin, Alemania.

Cruz-Suárez, L.E., D. Ricque-Marie, M. Tapia-Salazar, I.M. McCallum \& D. Hickling. 2001. Assessment of differently processed feed pea (Pisum sativum) meals and canola meal (Brassica sp.) in diets for blue shrimp (Litopenaeus stylirostris). Aquaculture 196: $87-104$.

Cruz-Suárez, L.E., M. Tapia-Salazar, D. Villarreal-Cavazos, J. Beltran-Rocha, M.G. Nieto-López, A. Lemme \& D. Ricque-Marie. 2009. Apparent dry matter, energy, protein and amino acid digestibility of four soybean ingredients in white shrimp Litopenaeus vannamei juveniles. Aquaculture 292: 87-94.

Dale, N. 1995. Ingredient analysis table. Feedstuffs Reference Issue. Vol. 76: 27-28. Minnetonka, Minesota, EEUU.

Davis, A., T.M. Samocha \& R.A. Bullis. 2004. Practical diets for Litopenaeus vannamei (Boone, 1931) working towards organics and/or all plant production diets, p. 202-214. In L.E. Cruz, D. Ricque, M.G. Nieto, D.A. Villarreal, U. Scholtz \& M.L. González (eds). Avances en Nutrición Acuícola VII. Memorias del Séptimo Simposium Internacional de Nutrición Acuícola. 16 al 19 de noviembre. Hermosillo, Sonora, México.

DEGUSSA, 2006. AminoDat ${ }^{\circledR}$ V. 3.0, Gold CD-Rom. México, Distrito Federal México.

Divakaran, S., M. Velasco, E. Beyer, I. Foster \& A. Tacon. 2000. Soybean meal apparent digestibility for Litopenaeus vannamei, including a critique of methodology, p. 267-276. In: L.E. Cruz -Suárez, D. Ricque-Marie, M. Tapia-Salazar, M.A. Olvera-Novoa \& R. CiveraCerecedo (eds.). Avances en Nutrición Acuícola V. Memorias del V Simposium Internacional de Nutrición Acuícola. 19-22 Noviembre, Mérida, Yucatán, México.
Fagbenro, O.A. \& S.J. Davies. 2001. Use of soybean flour (dehulled, solvent-extracted soybean) as a fish meal substitute in practical diets for African cat fish, Clarias gariepinus (Burchell 1822): growth, feed utilization and digestibility. J. Appl. Ichthyol. 17: 64-69.

Forster, I.P. \& W.D. Dominy. 2006. Efficacy of three methionine sources in diets for pacific white shrimp, Litopenaeus vannamei. J. World Aquac. Soc. 37: 474-480.

Furukawa, H. \& H. Tsukahara. 1966. On the acid digestion method for the determination of chromium oxide as an index substance in the study of digestibility of fish fed. Bull. Jap. Soc. Sci. Fish. 32: 502-506.

Gatlin III, D.M., F.T. Barrows, P. Brown, K. Dabrowski, T.G. Gaylord, R.W. Hardy, E. Herman, G. Hu, A. Krogdahl, R. Nelson, K. Overturf, M. Rust, W. Sealey, D. Skonberg, E.J. Souza, D. Stone, R. Wilson \& E. Wurtele. 2007. Expanding the utilization of sustainable plant products in aquafeeds: a review. Aquac. Res. 38: 551-579.

Guimarães, I.G., L.E. Pezzato, M.M. Barros \& L.Tachibana. 2008. Nutrient digestibility of cereal grain products and by-products in extruded diets for Nile Tilapia. J. World Aquac. Soc. 39: 781-788.

Hernández, C., M.A. Olvera-Novoa, K. Aguilar-Vejar, B. González-Rodríguez \& I. Abdo de la Parra. 2008. Partial replacement of fish meal by porcine meat meal in practical diets for pacific white shrimp Litopenaeus vannamei. Aquaculture 277: 244-250.

Kaur, V.I. \& P.K. Saxena. 2005. Incorporation of maize gluten in supplementary feed and its impact on growth and flesh quality of some carps. Aquac. Int. 13: $555-573$.

Lazo, J.P., R. Romaire \& R. Reigh. 1998. Evaluation of three in vitro enzyme assays for estimating protein digestibility in the pacific white shrimp Penaeus vannamei. J. World Aquac. Soc. 29: 441-450.

Lim, C. \& W. Dominy. 1990. Evaluation of soybean meal as a replacement for marine animal protein in diets for shrimp (Penaeus vannamei). Aquaculture 87: 53-63.

Liou, Ch.H., S.C. Lina \& J.H. Cheng. 2005. Urinary amino acid excretion by marine shrimp, Penaeus monodon, in response to orally administrated intact protein and crystalline amino acids. Aquaculture 248: 35-40.

Mariscal-Landín, G., J.H. Avellaneda, T.C. Reis de Souza, A. Aguilera, G.A. Borbolla \& B. Mar. 2004. Effect of tannins in sorghum on amino acid ileal digestibility and on trypsin (E.C.2.4.21.4) and chymotrypsin (E.C.2.4.21.1) activity of growing pigs. Anim. Feed Sci. Technol. 117: 245-264. 
McKevith, B. 2004. Nutritional aspects of cereals. British Nutrition Foundation, Londres, Reino Unido. Nutrition Bulletin 29: 111-142.

Molina, C. 2009. Evaluación del gluten de maíz como fuente reemplazante de la harina de pescado en dietas para el camarón juvenil Litopenaeus vannamei. CENAIM INFORMA, Boletín Interactivo No. 106. Ecuador. (Consultado 20Septiembre 2009, ww.cenaim.espol. edu.ec/publicaciones/quincenal/bquinc106.

Mu, Y.Y., T.J. Lam, J.J. Guo \& K.F. Shim. 2000. Protein digestibility and amino acid availability of several protein soureces for juvenile Chinese hairy crab Eriocheir sinensis H. Milne-Edwards (Decapoda, Grapsidae). Aquac. Res. 31: 757-765.

NRC. 1994. Nutrient Requirements of Poultry. National Research Council. National Academy, Washington, Columbia, EEUU.

NRC. 1998. Nutrient Requirements of Swine. National Research Council. National Academy, Washington, Columbia, EEUU.

Rawles, S.D. \& D.M. Gatlin III. 2000. Nutrient Digestibility of common feedstuffs in extruded diets for sunshine bass Morone chrysops $\bigcirc^{7}$ x M. saxatilis $\bigcirc$. J. World Aquac. Soc. 31: 474-480.

Rivas-Vega, M.E., O. Rouzaud-Sandez, M.G. Salazar-Garcia, J.M. Ezquerra-Brauer, E. Goytortua-Bores \& R. Civera-Cerecedo. 2009. Physicochemical properties of cowpea (Vigna unguiculata L. Walp.) meals and their apparent digestibility in white shrimp (Litopenaeus vannamei Boone). Hidrobiológica 19: 15-23.

SAS/STAT®. 1999. User's Guide, Version 8, SAS Institute Inc. Cary, Carolina del Norte, EEUU.

Sibbald, I.R. 1987. Estimation of available amino acids in feedingstuffs for poultry and pigs: a review with emphasis on balance experiments. Can. J. Anim. Sci. 67: 221-300.

Steel, R.G.D. \& J.H. Torrie. 1996. Bioestadística: Principios y procedimientos. Mc Graw-Hill. México, Distrito Federal, México.

Sudaryono, A., E. Tsvetnenko \& L.H. Evans. 1999. Replacement of soybean meal by lupin meal in practical diets for juvenile Penaeus monodon. J. World Aquac. Soc. 30: 46-57.

Tacon, A. 1989. Nutrición y alimentación de peces y camarones cultivados. Manual de Capacitación. Proyecto AQUILA II. Documento de Campo No. 4, GCP/ RLA/102/ITA. Programa Cooperativo Gubernamental FAO-Italia.
Tacon A.G.J., J.J. Cody, L.D. Conquest, S. Divakaran, I.P. Forster \& O.E. Decamp. 2002. Effect of culture system on the nutrition and growth performance of Pacific white shrimp Litopenaeus vannamei (Boone) fed different diets. Aquac. Nut. 8: 121-137.

Terrazas-Fierro, M.M., E. Ávila-González, M. Cuca-García \& H. Nolasco-Soria. 2005. Efecto de la incorporación de harina de pescado con distinto grado de cocción a dietas para pollos de engorda formuladas a un perfil de aminoácidos digestibles. Téc. Pec. Méx. 43: $297-$ 308 .

Thompson, K.R., S.D. Rawles, L.S. Metts, R. Smith, A. Winsatt, A.L. Gannam, R.G. Twibell, R.B. Johnson, Y.J. Brady \& C.D. Websteri. 2008. Digestibility of dry matter, protein, lipid, and organic matter of two fish meals, two poultry by-product meals, soybean meal, and distiller's dried grains with solubles in practical diets for Sunshine Bass, Morone chrysops 3 M. saxatilis. J. World Aquac. Soc. 39: 352-363.

Tibbetts, S.M., P.L. Santosh \& J.E. Milley. 2004 . Apparent digestibility of common feed ingredients by juvenile haddock, Melanogrammus aeglefinus L. Aquac. Res. 35: 643-651.

Wathelet, B. 1999. Nutritional analysis for proteins and amino acids in beans (Phaseolus sp.). Biotechnol. Agron. Soc. Environ. 3: 197-200.

Wilson, R.P. 2003. Amino Acid Requirements of Finfish and Crustaceans, p. 427-448. In J.P.F. D'Mello. Amino Acids in Animal Nutrition. CAB. Wallingford, Oxon, Reino Unido.

Wu, G., P.K. Davis, N.E. Flynn, D.A. Knabe \& J.T. Davidson. 1997. Endogenous synthesis of arginine plays an important role in maintaining arginine homeostasis in postweaning growing pigs. J. Nutr. 127: 2342-2349.

Yang, Q., X. Zhou, Q. Zhou, B. Tan, S. Chi, \& X. Dong. 2009. Apparent digestibility of selected feed ingredients for white shrimp Litopenaeus vannamei, Boone. Aquac. Res. 40: 1-9.

Yu, Y. 2006. Use of poultry by-product meal, and meat and bone meal. In Asian Aquafeeds: Current developments in the aquaculture feed industry. W.K. Ng \& C.K. Ng (eds.). Malaysian Fisheries Soc. Occasional Publication No. 13, Kuala Lumpur, Malasia.

Yu, Y. 2006a. Rendered products in shrimp aquaculture feeds. In D. Meeker (ed.). Essential Rendering. National Renderers Association, Kirby. Arlington, Virginia, EEUU. 